\title{
More than meets the eye: Split-second social perception
}

\author{
Jonathan B. Freeman ${ }^{1 *}$ and Kerri L. Johnson ${ }^{2}$ \\ ${ }^{1}$ New York University \\ ${ }^{2}$ University of California, Los Angeles \\ *Correspondence: jon.freeman @ nyu.edu (J. B. Freeman)
}

Keywords: person perception, top-down effects, face processing, stereotypes, neuroimaging, computational models

\begin{abstract}
Recent research suggests that visual perception of social categories is shaped not only by facial features but also higher-order social cognitive processes (e.g., stereotypes, attitudes, goals). Building on neural computational models of social perception, we outline a perspective of how multiple bottom-up visual cues are flexibly integrated with a range of top-down processes to form perceptions, and we identify a set of key brain regions involved. During this integration, "hidden" social category activations are often triggered, which temporarily impact perception without manifesting in explicit perceptual judgments. Importantly, these hidden impacts and other aspects of the perceptual process predict downstream social consequences-from politicians' electoral success to a number of evaluative biases-independent of the outcomes of that process.
\end{abstract}

(C) 2016. This manuscript version is made available under the Elsevier user license http://www.elsevier.com/open-access/userlicense/1.0/ 


\section{Visual Perception of Social Categories}

Based upon the mere glimpse of another individual, knowledge about that person's gender, race, and other social categories seems to spring to mind spontaneously. The visual construal of social categories feels instantaneous and immediate, as if it were a direct product of "reading" visible facial cues. This experience of social perception aligns with early research on the topic, which emphasized its automatic, immediate, and unavoidable nature [1-3].

A considerable body of research has shown that multiple social categories are perceived spontaneously when we encounter another individual [2]. Consequently, early work in the field of social psychology focused on either the inevitability of categorization or on its varied downstream consequences. Meanwhile, research in the cognitive, neural, and vision sciences sought to uncover the determinant cues and basic mechanisms driving perceptions of facial stimuli. Recently, a unified social vision approach has emerged [4-6], in which the visual aspects of social perception are theoretically and empirically integrated with the products that follow (see Glossary). This approach moves beyond the historic disciplinary divide in which each level of analysis was studied in relative isolation. As such, the social vision approach has afforded novel insights into how we form our initial perceptions of others, revealing important complexity that occurs prior to a stabilized judgment. Moreover, such dynamics during the process of forming categorical percepts of other people have recently been shown to carry downstream social consequences_-predicting politicians' electoral success, producing gender bias, and even eliciting prejudice against racial or sexual minorities.

Because downstream interpersonal consequences typically took center-stage in social perception research, a general feed-forward approach was generally assumed (Fig. 1A). As such, perceptual cues were presumed to activate a single, dominant social category representation, which in turn elicited related stereotypes, attitudes, and goals, thereafter impacting evaluative judgments and interpersonal behavior [1-3]. In this article, we offer a more comprehensive perspective in which social perception is characterized as the dynamic integration of both visual processing of facial features and higher-order social cognitive processes (e.g., stereotypes, attitudes, goals) that were historically probed only as a "downstream" product of social perception.

One formalization of this approach is the Dynamic Interactive (DI) Model, a neural computational model of social perceptions [4] (Fig. 1A). The DI Model treats an initial percept 
as an automatic, yet gradual negotiation between the multiple visual features inherent in a target of perception and the prior knowledge or high-level cognitive states that a perceiver brings to the perceptual process (e.g., stereotypes, attitudes, goals). Thus, initial perceptions are not discrete "read outs" of visual features; they evolve over hundreds of milliseconds and may be dynamically shaped by context and higher-order social cognition. Certain models of social reasoning and high-level impression formation have long considered similar integrative processes and feedback influences [7], but these treated initial social perceptions as theoretical givens and as input into the model. In contrast, the DI Model aims to explain and predict the split-second perception itself [4].

\section{Malleability and Bias of Initial Perceptions}

Perceivers are highly sensitive not only to faces' discrete social categories but also to within-category variation in cues (e.g., gender or racial typicality). Such variation impacts initial categorizations [8,9], stereotype activation [10,11] and application [12], evaluative judgments [13], and other interpersonal behaviors. For instance, more prototypically Black faces more strongly activate Black-related stereotypes, and this portends harsher criminal sentences [14] including capital punishment [15]. Similarly, higher levels of gender typicality impact sex categorizations [16], activate stronger gender-based stereotypes [11], and elicit favorable social evaluations [17]. For example, American female politicians with more feminine facial features enjoy higher levels of electoral success, particularly for elections held in conservative American states $[18,19]$. In general, such typicality biases persist even when controlling for evaluative factors such as perceived attractiveness and competence [e.g., 18]. Moreover, implicit stereotypes and attitudes exist independent of a perceiver's own gender, race, or other category memberships because they tend to be learned, though not necessarily endorsed, by all members within a society [20].

Although downstream evaluative biases commonly stem from negative attitudes related to a perceived category (or deviations from that category), recent work reveals that some biases are rooted in the initial perception of the category itself. Prejudice against mixed-race individuals is predicted by a more unstable perceptual experience during the processing of mixed-race faces - one that involves abruptly shifting race-category representations - an effect that is stronger in White observers who have little exposure to Black individuals [21]. Similarly, 
prejudice against sexual minorities occurs in part because gender-atypical faces tend to be categorized as gay due to stereotypical associations [22]. That said, the effect of gay categorization on negative evaluations is statistically mediated by greater difficulty in perceiving the face's gender [23]. Finally, systematically exposing observers to either masculine or feminine features in visual-adaptation paradigms produces visual aftereffects that shift perceived norms for what is regarded as "typical" and "atypical", thus also altering social evaluations. For instance, exposing observers to more extreme levels of masculine features adapts the visual system such that a subsequent face requires more masculinity to be perceived as male. Faces that deviate from this new male norm (which now appear more feminine to the perceiver) are more difficult to categorize, in turn leading them to be evaluated more negatively [24]. Thus, some forms of prejudice may originate in aspects of the initial perceptual process itself, such as processing ease or stability, independent of the categorical outcomes of that process [25]. Social perceptions are not only sensitive to bottom-up cues originating in the target of perception, but also to top-down factors harbored within perceivers, such as stereotypes [26,27], goals [28-30], and prior person-knowledge [31,32]. Which race-category is initially perceived from a face is influenced by prior racial labels [33], stereotypes [26,34], social dominance [35], visual context [36,37], and political and economic factors [30,38]. Perceptions of a face's race, gender, and emotion categories are biased by stereotypical expectations, and these biases manifest at relatively early levels of visual representation in the brain [39]. Higher-order goals, such as self-protection motives, can also bias perceptions, leading ambiguous targets to more readily be perceived as male or as Black due to associations with a potential to harm [17].

Thus, extant work suggests initial perceptions are not solely determined by visible cues, thereafter driving evaluation and behavior (Fig. 1). Instead, perceivers are sensitive to subtle variation in facial features that impact the perceptual process, and perceptual experiences themselves can affect subsequent interpersonal processes independent of explicit categorizations. In addition, social perceptions are malleable and influenced by feedback from higher-order social cognitive processes traditionally considered "downstream” from initial perceptions. Together, these findings suggest initial perceptions involve a rapid and flexible integration of multiple bottom-up visual cues and top-down processes. Next, we delve into the underlying mechanisms that permit such integration in social perception. 


\section{A Landscape of Hidden Social Categories}

Social targets inhabit multiple categories simultaneously (e.g., young Asian female). Classic work suggested this "multiple category problem" is solved by only one dominant category coming to the fore while others are actively suppressed [40,41]. Recent research, however, reveals that multiple categories both across and within dimensions co-exist throughout processing $[4,42]$. Although perceivers' judgments stabilize on a single categorization rapidly, numerous "hidden" categories are concurrently activated - even though these parallel activations may not be apparent in explicit behavioral responses. These concurrent activations are elicited by both bottom-up cues that originate in the target of perception (e.g., subtle feminine cues on a male face partially activate the female category [43]) and top-down factors harbored within the perceiver (e.g., stereotypical expectations that Black individuals are hostile partially activate the angry category even when not displaying any anger $[39,44])$. Although bottom-up and top-down processes dynamically impinge on social perceptions, competition between category alternatives via lateral inhibition typically clears such "incorrect" hidden activations from processing [4] (Fig. 1)

Evidence for hidden social category activations has typically utilized computer mousetracking paradigms (Box 1). Participants rapidly categorize faces by moving the computer cursor from the bottom-center of the screen into one of two category responses in either top corners. The premise is that the hand in motion reveals "mind in motion" and the real-time unfolding of cognitive processes. Such mouse-tracking paradigms have now been leveraged in many domains across the cognitive sciences [45-47]. Early studies explored natural withincategory variation in facial cues and the hidden activations they trigger. No face is a perfect prototype of a given category, instead inhabiting middle-points along the pertinent socialperceptual continua (e.g., gender, race), and this subtly is revealed in mouse trajectories. For instance, when categorizing a face bearing subtle cues of the opposite gender, participants' mouse trajectories exhibited a partial attraction toward the opposite gender-category response before ultimately selecting the correct category [16]. This provided evidence that an alternate social category (e.g., female) became partially activated, even though it did not manifest in the explicit perceptual judgment (e.g., male). Once activated, these partial-parallel activations of social categories (e.g., female) cascade into the partial-parallel triggering of associated stereotypes (e.g., caring) [11] (Fig. 1). Importantly, these temporary activations also predict 
meaningful downstream consequences and real-world outcomes, independent of category membership itself (Fig. 2A)

The hidden triggering of social categories has now been observed during perceptions of gender, race, age, emotion, and sexual-orientation categories, when specific facial cues resemble an alternate category $[9,16,44,48,49]$. These concurrent activations are also elicited by the topdown influence of stereotypes [26,44], prior knowledge [48], and expectations due to context [37] (Box 2). For instance, due to stereotypical associations between racial categories and social status, participants' hand trajectories were initially attracted to the Black response when categorizing White faces surrounded by low-status attire (e.g., janitor uniform), and attracted to the White response when categorizing Black faces surrounded by high-status attire (e.g., business suit); these stereotypic biases were exacerbated as race became more ambiguous, as bottom-up ambiguity opens the door wider to top-down influences [26] (Fig. 2B).

These concurrent activations differ not only in their strength, but also in their qualitative dynamics. When the environmental context surrounding a target is more consistent with an alternate category (e.g., a White face in an Asian-related scene), the alternate category became partially activated, as indicated by a temporary partial attraction toward the alternative (e.g., Asian). Interestingly, this temporary activation occurred significantly earlier among perceivers from more collectivist, 'high-context' cultures than among perceivers from more individualist, 'low-context' cultures (Box 1, Fig. I). This finding implies that a cultural preparedness to process context shapes the timing of its impact on perception [37]. Additional perceiver characteristics, such as exposure to different racial groups, influences the stability with which racial categories activate during initial perception. For Whites living in neighborhoods with lower interracial exposure, racially-ambiguous faces elicited co-activations of the White and Black categories that competed in a more unstable fashion, as indicated by abrupt, back-and-forth shifting between category responses and higher velocity in hand motion (Figs. 2C and 3). In turn, this more unstable perceptual experience of racial ambiguity predicted a higher prejudice against mixedrace individuals and mediated the effect of having lower interracial exposure on this prejudice. Beyond interracial exposure, hidden impacts on perception and temporary category activations are sensitive to a number of individual differences, including the strength of stereotypic associations [39,50], attitudes [44], cultural differences [37], and neurodevelopmental disorders [51] — often without any impact on explicit judgments or ultimate perceptual outcomes. 


\section{Inherent Intersection of Social Categories}

Social category dimensions were historically considered to be processed in isolation, but recent studies have demonstrated their inherently intersectional nature. For example, the DI Model (Fig. 1) predicts that multiple social categories will be perceived interdependently when stereotypes related to two ostensibly unrelated categories overlap. This is because the processing of one category dimension (e.g., sex) will activate stereotypes that in turn bias the perception of other category dimensions (e.g., race) while perception is still unfolding. To the extent that two social categories (e.g., male and Black) share stereotypes (e.g., 'aggressive'), those social categories themselves will become inextricably linked, down to a perceptual level.

Accordingly, recent studies have discovered that race categories bias gender perception and vice-versa, such that Black female faces partially activate the male category and Asian male faces partially activate the female category during face perception. Because These "race is gendered" effects are exacerbated for perceivers holding stronger overlapping stereotypes between Black and male categories, and Asian and female categories [50,52]. These perceptual biases also have lasting impacts, predicting leadership selection or interracial dating [53]. Other categories also become perceptually entangled with race and gender, such as emotion. Stereotypically, men and Black individuals are both associated with expressing anger, while women are associated with expressing joy; consequently, emotion perception is biased in a consistent fashion $[34,54]$.

In a set of fMRI studies [39], participants viewed faces varying by gender, race, and emotion, and additionally completed post-scan mouse-tracking tasks (Fig. 4). The extent of a participant's conceptual (i.e., stereotype) overlap between pairs of categories was also assessed. Overall, mouse trajectories showed intersectional bias toward a related, yet orthogonal category: male and Black toward anger, female toward happy, Black toward male, and Asian toward female. Despite these overall tendencies, participants' own unique stereotype overlaps between categories predicted the extent of those stereotypes' hidden impact on perceptions. At the neural level, these stereotypically-biased effects on perception were reflected in the similarity of the categories' multi-voxel representations in the FG and OFC (Fig. 1B). For instance, a stronger attraction to select the 'angry' response for Black faces predicted greater neural-pattern similarity between angry and Black category patterns in these regions. This held true even when stimuli 
were matched on low-level visual properties and after controlling for several bottom-up visual models (e.g., pixel-based similarity or models of ventral-visual processing), ensuring that such perceptual biases were not due to mere physical confounds (Fig. 4). That these stereotype-driven biases on perception manifest in the representational structure of visual face-processing regions (FG) supports the notion that top-down feedback from social cognitive processes can reach relatively low levels of perceptual representation. Finally, additional studies have found that brain regions associated with conflict-monitoring and inhibition may also be involved in helping correct these temporary biases during perception (e.g., attraction to 'angry' for a non-angry Black face) once they become activated [44].

\section{A Neural Network for Flexible Social Perception}

As outlined above, recent behavioral studies point to a split-second social perception process that involves flexible integration of multiple bottom-up visual cues and top-down social cognitive factors - a process that often involves the hidden triggering of multiple category activations that impact perception. At the neural level, extant research suggests a network of three regions is important for this process, the fusiform gyrus (FG), orbitofrontal cortex (OFC), and anterior temporal lobe (ATL), which are all densely interconnected [55,56] (Fig. 1B). We focus on these regions for their specific role in integrating bottom-up and top-down information in split-second perception of another's face, but clearly numerous other regions participate in additional components of social perception more broadly.

The FG is centrally involved in face perception, and it is highly responsive to face and face-like stimuli [57]. Whereas extrastriate areas are sensitive to a face's physical variation, the FG is more sensitive to a face's abstract, categorical distinctions [58]. Indeed, the FG has been shown to be specifically involved in representing social-categorical information from faces. For instance, activity in the FG is sensitive to within-category variation in social categories [59], and multi-voxel patterns within the region reliably distinguish gender, race, and emotion categories [60-63]. Although bottom-up visual cues are clearly the driving input to the region, FG activity is also malleable to a number of higher-order social cognitive processes, including stereotypes [39], goals [64] and implicit biases [63,65,66]. Indeed, recent studies combining MVPA with mousetracking suggest that hidden social category activations driven by such higher-order processes impact initial perceptions via biased representations in the FG [39] (Box 2). 
Although models of face perception recognize an extensive network of downstream regions involved in subsequent processing once a face is initially analyzed [67], these regions are generally not regarded as participating in the initial face-perceptual process. However, recent evidence suggests the OFC and ATL may be involved in this process, helping to situate the face within a larger social context and align perceptual representations with higher-order processes. Indeed, the notion that top-down feedback from higher-order neural systems may modulate lower-level visual processing is hardly new, and top-down effects have been observed as early as primary visual cortex (V1) for simple visual stimuli [68-70]. In cases of face stimuli, a primary target of top-down feedback is the FG [71,72].

Although in theory any number of social cognitive processes may constrain initial perceptions (e.g., stereotypes, attitudes, goals), the top-down impact of stereotypes has the strongest support at a mechanistic level. Stereotypes are merely semantic associations activated by social categories, which are theorized to automatically trigger expectations and predictions [4]; more generally, semantic contexts have long been known to constrain perception of objects or words [73,74]. Indeed, models such as the DI Model [4,21,26] (Fig. 1) find that stereotypes' top-down effects on face perception occur through computational principles that are similar to classic context effects on word perception [73]. As implicit expectations generated during perception $[10,11]$, stereotypes influence face perception and context influences object or word perception in an analogous manner (Fig. 5), supporting the possibility of domain-general mechanisms.

Considering this similarity with other context effects, numerous studies have implicated the OFC in modulating visual-object recognition via top-down expectations activated by contextual or associative cues $[75,76]$. Top-down perceptual 'priors' from the OFC may have a modulatory role on ventral-visual representation [72,74], and expectations about face stimuli in particular enhance top-down effective connectivity from the OFC to the FG [71,72]. MEG studies show that activity related to successful object recognition is present in the OFC 50-85 ms earlier than in ventral-visual object-processing regions, consistent with the OFC's ability to exert top-down modulation on perceptual regions [77]. The OFC has additionally been linked to the integration of face's social category cues with context-based expectations about those cues [75], the retrieval of person knowledge [78], and the accessing of stereotypes $[79,80]$. 
The OFC also responds to within-category variation in gender and race [36,59], and recent work found that neural patterns in the $\mathrm{OFC}$ and FG exhibited a representational structure of faces' multiple social categories (gender, race, and emotion) that was biased by stereotypical associations [39]. For example, when categorizing Black faces that exhibited no actual angry cues, the degree of a participant's temporary attraction to select the 'angry' response predicted greater neural-pattern similarity between Black and angry patterns in these regions [39] (Fig. 4). One possibility is that, in response to a face, social-conceptual associations are automatically retrieved (e.g., Black-angry), in turn leading the OFC to implement a top-down prediction of 'angry' that is then imposed on FG representation. Thus, recent studies suggest the OFC plays an important role in initial social perceptions, possibly by implementing visual predictions based on stereotypes, person-knowledge, or other socially-relevant expectations, to then sensitize perceptual regions (such as the FG) to fall in line with those predictions.

Although the OFC may implement visual predictions, it likely coordinates with the ATL to access social-conceptual associations that form those predictions. The ATL is consistently activated during the storage and retrieval of semantic associations, and one popular theory argues for its role as an amodal 'semantic hub' [81]. However, the ATL exhibits selective responses to social as opposed to non-social concepts and associations, such as person-knowledge and stereotypes $[60,82,83]$, leading some to argue for its selective role in the processing of socialsemantic knowledge [82]. It also commonly activates in response to faces, especially when associated with prior knowledge [82,84], and monkey studies show that ATL neurons rapidly generate associations between face stimuli and related abstract knowledge [85]. Demonstrating a causal role in activation of social-conceptual associations, temporarily disrupting ATL activity decreases stereotypes' associative strength (e.g., Arab = terrorist), as measured by an Implicit association test (IAT); however, no such decrease is observed for non-social associations [86]. Such studies support ATL involvement in accessing a diverse range of social-conceptual information, which may then be used by the OFC for visual predictions that in turn modulate FG face processing.

Taken together, emerging research suggests a key network involving the FG, OFC, and ATL in integrating bottom-up facial cues with top-down socially-relevant expectations or socialconceptual information to form initial perceptions of others. 


\section{Concluding Remarks}

Traditional approaches have emphasized that visual construal of others' social categories reflects an automatic "read-out" of facial features, which thereafter unleashes a linear chain of events from initial perceptions up to higher-order processes. In light of current evidence, here we argue that initial social perceptions are, in fact, hardly "initial". They reflect a dynamic cascade of interactive influences, wherein factors that were long presumed to be "downstream" products of social perception, such as stereotypes, attitudes, and goals, constrain initial perceptions. In this process, bottom-up visual cues inherent to targets and a range of top-down social cognitive processes harbored within perceivers are rapidly integrated to form split-second perceptions. As this integration unfolds, multiple "hidden" category activations may impact perception without being observed in explicit perceptual judgments. Although quickly cleared from processing, these hidden perceptual impacts and other aspects of the initial perceptual process can linger, driving important downstream social consequences, independent of the outcomes of that process. This overall perspective is consistent with neural computational models of social perception (Fig. 1) and points to a key network comprised of the FG, OFC, and ATL in helping form these perceptions. In such a network, perceptual representations of another's face available in the FG may be routinely modulated by top-down visual predictions of the OFC, which are guided by social-conceptual associations retrieved in the ATL.

Together, the work presented here suggests that the nature of social perception may be far more bidirectional than typically considered. Not only are visual perceptions of other people malleable to higher-order social cognition, but the dynamics of these perceptions, in turn, may uniquely impact higher-order processes and bear downstream effects on social evaluation and behavior. However, such research is only emerging and many questions remain (see Outstanding Questions). Moreover, this recent work has predominantly focused on perceiving social categories, but in the future this perspective can be leveraged for other instances of social perception, such as face-based perceptions of traits or mental states. Additionally, although our focus has been on perceptions via facial cues, perceptions via bodily or vocal cues show similar integrative effects, and how these function together warrants further investigation $[27,87,88]$. We are hopeful that advances in understanding split-second social perception will be furthered from burgeoning social vision approaches and an increasing cross-talk between social psychology and the cognitive, neural, and vision sciences. 


\section{Author's Note}

This work was funded in part by research grant NSF-BCS-1423708 (J.B.F). 


\section{References}

1 Brewer, M.B. (1988) A dual process model of impression formation. In A Dual-Process Model of Impression Formation: Advances in Social Cognition (Srull, T.K. and Wyer, R.S., eds), pp. 136, Erlbaum

2 Macrae, C.N. and Bodenhausen, G.V. (2000) Social cognition: Thinking categorically about others. Annual Review of Psychology 51, 93-120

3 Fiske, S.T. and Neuberg, S.L. (1990) A continuum model of impression formation from category-based to individuating processes: Influences of information and motivation on attention and interpretation. Advances in Experimental Social Psychology 23, 1-74

4 Freeman, J.B. and Ambady, N. (2011) A dynamic interactive theory of person construal. Psychological Review 118, 247-279

5 Adams, R.B., et al. (2011) The Science of Social Vision. Oxford University Press 6 Balcetis, E. and Lassiter, D. (2010) The Social Psychology of Visual Perception. Psychology Press

7 Kunda, Z. and Thagard, P. (1996) Forming impressions from stereotypes, traits, and behaviors: A parallel-constraint-satisfaction theory. Psychological Review 103, 284-308

8 Freeman, J.B., et al. (2008) Will a category cue attract you? Motor output reveals dynamic competition across person construal. Journal of Experimental Psychology: General 137, 673-690 9 Freeman, J.B., et al. (2010) Continuous dynamics in the real-time perception of race. Journal of Experimental Social Psychology 46, 179-185

10 Mason, M.F., et al. (2006) On construing others: Category and stereotype activation from facial cues. Social Cognition 24, 540-562

11 Freeman, J.B. and Ambady, N. (2009) Motions of the hand expose the partial and parallel activation of stereotypes. Psychological Science 20, 1183-1188

12 Blair, I.V., et al. (2002) The role of Afrocentric features in person perception: Judging by features and categories. Journal of Personality and Social Psychology 83, 5-25

13 Livingston, R.W. and Brewer, M.B. (2002) What are we really priming? Cue-based versus category-based processing of facial stimuli. Journal of Personality and Social Psychology 82, 518

14 Blair, I.V., et al. (2004) The influence of Afrocentric facial features in criminal sentencing. Psychological Science 15, 674-679 
15 Eberhardt, J.L., et al. (2006) Looking deathworthy perceived stereotypicality of black defendants predicts capital-sentencing outcomes. Psychological Science 17, 383-386 16 Freeman, J.B., et al. (2008) Will a category cue attract you? Motor output reveals dynamic competition across person construal. Journal of Experimental Psychology: General 137, 673-690 17 Johnson, K.L., et al. (2015) Emergent research in social vision: An integrated approach to the determinants and consequences of social categorization. Social and Personality Psychology Compass 9, 15-30

18 Hehman, E., et al. (2014) Early processing of gendered facial cues predicts the electoral success of female politicians. Social Psychological and Personality Science 5, 815-824

19 Carpinella, C.M., et al. (2015) The Gendered Face of Partisan Politics: Consequences of Facial Sex Typicality for Vote Choice. Political Communication, 1-18

20 Nosek, B.A., et al. (2002) Harvesting implicit group attitudes and beliefs from a demonstration web site. Group Dynamics: Theory, Research, and Practice 6, 101

21 Freeman, J.B., et al. (in press) A perceptual pathway to bias: Interracial exposure reduces abrupt shifts in real-time race perception that predict mixed-race bias. Psychological Science 22 Freeman, J.B., et al. (2010) Sexual orientation perception involves gendered facial cues. Personality and Social Psychology Bulletin 36, 1318-1331

23 Lick, D.J. and Johnson, K.L. (2013) Fluency of visual processing explains prejudiced evaluations following categorization of concealable identities. Journal of Experimental Social Psychology 49, 419-425

24 Lick, D.J. and Johnson, K.L. (2014) Recalibrating gender perception: Face aftereffects and the perceptual underpinnings of gender-related biases. Journal of Experimental Psychology: General 143, 1259

25 Lick, D.J. and Johnson, K.L. (2015) The Interpersonal Consequences of Processing Ease Fluency as a Metacognitive Foundation for Prejudice. Current Directions in Psychological Science 24, 143-148

26 Freeman, J.B., et al. (2011) Looking the part: Social status cues shape race perception. PLoS ONE 6, e25107

27 Johnson, K.L., et al. (2011) He throws like a girl (but only when he's sad): Emotion affects sex-decoding of biological motion displays. Cognition 119, 265-280 
28 Caruso, E.M., et al. (2009) Political partisanship influences perception of biracial candidates' skin tone. Proceedings of the National Academy of Sciences 106, 20168-20173

29 Ratner, K.G., et al. (2014) Visualizing minimal ingroup and outgroup faces: implications for impressions, attitudes, and behavior. Journal of personality and social psychology 106, 897 30 Krosch, A.R. and Amodio, D.M. (2014) Economic scarcity alters the perception of race. Proceedings of the National Academy of Sciences 111, 9079-9084

31 Freeman, J.B., et al. (2010) The neural origins of superficial and individuated judgments about ingroup and outgroup members. Human Brain Mapping 31, 150-159

32 Anderson, E., et al. (2011) The visual impact of gossip. Science 332, 1446-1448

33 Tskhay, K.O. and Rule, N.O. (2015) Semantic Information Influences Race Categorization From Faces. Personality and Social Psychology Bulletin, 0146167215579053

34 Hugenberg, K. and Bodenhausen, G.V. (2004) Ambiguity in social categorization: The role of prejudice and facial affect in race categorization. Psychological Science 15, 342-345

35 Ho, A.K., et al. (2013) Status boundary enforcement and the categorization of black-white biracials. J. Exp. Soc. Psychol. 49, 940-943

36 Freeman, J.B., et al. (2015) The neural basis of contextual influences on face categorization. Cerebral Cortex 25, 415-422

37 Freeman, J.B., et al. (2013) Influences of culture and visual context on real-time social categorization. Journal of experimental social psychology 49, 206-210

38 Krosch, A.R., et al. (2013) On the ideology of hypodescent: Political conservatism predicts categorization of racially ambiguous faces as Black. Journal of Experimental Social Psychology 49, 1196-1203

39 Stolier, R.M. and Freeman, J.B. (invited revision) Neural pattern similarity reveals the inherent intersection of social categories. Nature Neuroscience 40 Bodenhausen, G.V. and Macrae, C.N. (1998) Stereotype activation and inhibition. In Stereotype activation and inhibition, pp. 1-52, Lawrence Erlbaum Associates Publishers 41 Macrae, C.N., et al. (1995) The dissection of selection in person perception: Inhibitory processes in social stereotyping. Journal of Personality and Social Psychology 69, 397-407 42 Freeman, J.B., et al. (2013) Finger in flight reveals parallel categorization across multiple social dimensions. Social Cognition 31, 792-805 
43 Freeman, J.B. (2014) Abrupt category shifts during real-time person perception. Psychonomic bulletin \& review $21,85-92$

44 Hehman, E., et al. (2014) The neural basis of stereotypic impact on multiple social categorization. Neuroimage 101, 704-711

45 Freeman, J.B., et al. (2011) Hand in motion reveals mind in motion. Frontiers in Psychology 2,59

46 Song, J.H. and Nakayama, K. (2009) Hidden cognitive states revealed in choice reaching tasks. Trends in Cognitive Sciences 13, 360-366

47 Freeman, J.B. and Ambady, N. (2010) MouseTracker: Software for studying real-time mental processing using a computer mouse-tracking method. Behavior Research Methods 42, 226-241

48 Rule, N.O., et al. (2014) On the interactive influence of facial appearance and explicit knowledge in social categorization. European Journal of Social Psychology 44, 529-535 49 Freeman, J.B. and Ambady, N. (2011) Hand movements reveal the time-course of shape and pigmentation processing in social categorization. Psychonomic Bulletin and Review 18, 705-712 50 Johnson, K.L., et al. (2012) Race is gendered: How Covarying Phenotypes and Stereotypes Bias Sex Categorization. Journal of Personality and Social Psychology, doi: 10.1037/a0025335 51 Martens, M.A., et al. (2012) Continuous cognitive dynamics of the evaluation of trustworthiness in Williams syndrome. Frontiers in Psychology 3, 160 52 Carpinella, C.M., et al. (2015) Gendered facial cues influence race categorizations. Personality and Social Psychology Bulletin, 0146167214567153 53 Galinsky, A.D., et al. (2013) Gendered races implications for interracial marriage, leadership selection, and athletic participation. Psychological science, 0956797612457783 54 Hess, U., et al. (2004) Facial appearance, gender, and emotion expression. Emotion 4, 378388

55 Kringelbach, M.L. and Rolls, E.T. (2004) The functional neuroanatomy of the human orbitofrontal cortex: evidence from neuroimaging and neuropsychology. Progress in neurobiology 72, 341-372

56 Ding, S.L., et al. (2009) Parcellation of human temporal polar cortex: a combined analysis of multiple cytoarchitectonic, chemoarchitectonic, and pathological markers. Journal of Comparative Neurology 514, 595-623 
57 Kanwisher, N. and Yovel, G. (2006) The fusiform face area: a cortical region specialized for the perception of faces. Philosophical Transactions of the Royal Society of London. Series B: Biological Sciences 361, 2109-2128

58 Rotshtein, P., et al. (2005) Morphing Marilyn into Maggie dissociates physical and identity face representations in the brain. Nature Neuroscience 8, 107-113

59 Freeman, J.B., et al. (2010) The neural basis of categorical face perception: Graded representations of face gender in fusiform and orbitofrontal cortices. Cerebral Cortex 20, 13141322

60 Contreras, J.M., et al. (2013) Multivoxel patterns in fusiform face area differentiate faces by sex and race. PloS one 8, e69684

61 Wegrzyn, M., et al. (2015) Investigating the brain basis of facial expression perception using multi-voxel pattern analysis. Cortex

62 Ratner, K.G., et al. (2013) Is race erased? Decoding race from patterns of neural activity when skin color is not diagnostic of group boundaries. Social cognitive and affective neuroscience $8,750-755$

63 Brosch, T., et al. (2013) Implicit race bias decreases the similarity of neural representations of black and white faces. Psychological science 24, 160-166

64 Kaul, C., et al. (2013) Dynamic representations of race: processing goals shape race decoding in the fusiform gyri. Social cognitive and affective neuroscience

65 Kaul, C., et al. (2014) Dynamic representations of race: processing goals shape race decoding in the fusiform gyri. Social cognitive and affective neuroscience 9, 326-332

66 Van Bavel, J.J., et al. (2008) The neural substrates of in-group bias a functional magnetic resonance imaging investigation. Psychological Science 19, 1131-1139

67 Haxby, J.V., et al. (2000) The distributed human neural system for face perception. Trends in Cognitive Sciences 4, 223-233

68 Kok, P., et al. (2012) Less is more: expectation sharpens representations in the primary visual cortex. Neuron 75, 265-270

69 Gilbert, C.D. and Sigman, M. (2007) Brain states: Top-down influences in sensory processing. Neuron 54, 677-696

70 Li, W., et al. (2004) Perceptual learning and top-down influences in primary visual cortex.

Nature Neuroscience 7, 651-657 
71 Summerfield, C., et al. (2006) Predictive codes for forthcoming perception in the frontal cortex. Science 314, 1311-1314

72 Summerfield, C. and Egner, T. (2009) Expectation (and attention) in visual cognition. Trends in cognitive sciences $13,403-409$

73 McClelland, J.L. and Rumelhart, D.E. (1981) An interactive activation model of context effects in letter perception: Part 1. An account of basic findings. Psychological Review 88, 375407

74 Bar, M. (2004) Visual objects in context. Nature Reviews Neuroscience 5, 617-629

75 Freeman, J.B., et al. (2015) The neural basis of contextual influences on face categorization. Cerebral cortex 25

76 Bar, M., et al. (2006) Top-down facilitation of visual recognition. Proceedings of the National Academy of Sciences of the United States of America 103, 449-454

77 Kveraga, K., et al. (2007) Magnocellular projections as the trigger of top-down facilitation in recognition. Journal of Neuroscience 27, 13232-13240

78 Mitchell, J.P., et al. (2002) Distinct neural systems subserve person and object knowledge. Proceedings of the National Academy of Sciences of the United States of America 99, 1523815243

79 Milne, E. and Grafman, J. (2001) Ventromedial prefrontal cortex lesions in humans eliminate implicit gender stereotyping. The Journal of neuroscience : the official journal of the Society for Neuroscience 21, RC150

80 Knutson, K.M., et al. (2007) Neural correlates of automatic beliefs about gender and race. Human brain mapping 28, 915-930

81 Patterson, K., et al. (2007) Where do you know what you know? The representation of semantic knowledge in the human brain. Nature Reviews Neuroscience 8, 976-987

82 Olson, I.R., et al. (2012) Social cognition and the anterior temporal lobes: a review and theoretical framework. Social cognitive and affective neuroscience, nss119 83 Zahn, R., et al. (2007) Social concepts are represented in the superior anterior temporal cortex. . Proceedings of the National Academy of Sciences 104, 6430-6435 84 Ross, L.A. and Olson, I.R. (2012) What's unique about unique entities? An fMRI investigation of the semantics of famous faces and landmarks. Cerebral Cortex 22, 2005-2015 
85 Eifuku, S., et al. (2010) Neural correlates of associative face memory in the anterior inferior temporal cortex of monkeys. The Journal of Neuroscience 30, 15085-15096

86 Gallate, J., et al. (2011) Noninvasive brain stimulation reduces prejudice scores on an implicit association test. Neuropsychology 25, 185

87 Freeman, J.B. and Ambady, N. (2011) When two become one: Temporally dynamic integration of the face and voice. Journal of Experimental Social Psychology 47, 259-263

88 Campanella, S. and Belin, P. (2007) Integrating face and voice in person perception. Trends in Cognitive Sciences 11, 535-543

89 Spivey, M.J. and Dale, R. (2006) Continuous dynamics in real-time cognition. Current Directions in Psychological Science 15, 207-211

90 Freeman, J.B., et al. (2011) The real-time link between person perception and action: Brain potential evidence for dynamic continuity. Social Neuroscience 6, 139-155

91 Cisek, P. and Kalaska, J.F. (2005) Neural Correlates of Reaching Decisions in Dorsal Premotor Cortex: Specification of Multiple Direction Choices and Final Selection of Action. Neuron 45, 801-814

92 Sullivan, N., et al. (2015) Dietary self-control is related to the speed with which attributes of healthfulness and tastiness are processed. Psychological science 26, 122-134 93 Burton, A.M., et al. (2010) The Glasgow face matching test. Behavior Research Methods 42, 286-291 


\section{Glossary}

Social vision: An emerging field combining social psychology and vision science.

Feed-forward: A system or form of processing in which information moves in only one direction in a directed fashion without any feedback influences.

Stereotypes: Beliefs, expectations, or conceptual associations related to a social category, which may be positive, negative, or neutral, and which perceivers may implicitly hold without necessarily endorsing personally.

Attitudes: A positive or negative evaluation related to a social category (or person, object, thing, or event).

Computational model: A mathematical model used to study the behavior of complex systems. Neural computational models provide an algorithmic and process-level description of cognition using core principles of information processing in neural systems.

Visual aftereffects: Effects where prolonged exposure to stimuli on a visual dimension (e.g., orientation, color, facial gender) results in adaptation that biases perception of other stimuli on that dimension in a systematic fashion.

Ventral-visual processing: Ventral-visual processing or representation refers to the ventral pathway in the brain, extending from V1 through extrastriate areas and ventral regions of the temporal cortex, where visual stimuli are processed in detail and represented.

Electroencephalography (EEG): A noninvasive technique that measures electrical potentials in the brain, which provides high-resolution temporal information but fairly poor spatial resolution. Lateral inhibition: The process of an excited neuron, node, or representation diminishing the activity of its neighbors, creating a competition between lateral entities.

Attractors: A state toward which a dynamical system tends to evolve.

Extrastriate areas: Cortex next to V1, involved in early visual processing of stimuli.

Multi-voxel pattern analyses (MVPA) or multi-voxel representations: A multivariate approach to fMRI data where individual stimuli, conditions, or tasks are distinguished on the basis of local voxel response patterns.

Magnetoencephalography (MEG): A noninvasive technique that measures magnetic fields generated by electrical currents in the brain, which provides high-resolution temporal information and typically better spatial resolution than EEG (although worse than fMRI). 
Effective connectivity: Analyses that use statistical models of functional neuroimaging data to infer that the activity of one neural region is exerting a directed and causal influence on another neural region's activity.

Implicit association test (IAT): A reaction-time measure used to assess the strength of an individual's implicit associations in memory, independent of any conscious expression. 


\section{Box 1. Computer mouse-tracking paradigm}

Mouse-tracking tasks have been used across a variety of research areas in the cognitive sciences, including recent social perception research [45,47,89]. Although a two-choice paradigm is most popular, many other arrangements exist. In such tasks, the hand's trajectory is recorded during the real-time evolution of a behavioral response, providing high-fidelity information about a participant's tentative commitments to multiple resolve alternatives over time. This allows a discrete reaction time or button-press to be opened up into a continuous stream of cognitive output, which is warranted because motor dynamics are coextensive with cognitive dynamics [45]. For instance, in perceptual decision-making tasks, monkey neurophysiological work and human EEG studies demonstrate that, when required to make a hand-guided response, information continuously flows into the premotor cortex driving the motor response while the decision process evolves over time [90,91].

Participants begin a trial by clicking a button at the bottom-center of the screen, after which they are presented with the stimulus (e.g., a face). They then quickly move the cursor from the bottom-center of the screen to response options in either top corners (e.g., 'male' vs. 'female'). Despite their explicit selection, participants' hand movements can simultaneously exhibit a "hidden" partial attraction toward the unselected alternative (on the opposite side of the screen), indicating a competing, partial activation of that alternative. Numerous properties of these competitive motor dynamics are informative for understanding cognitive dynamics, including the magnitude of that activation and its real-time stability (Fig. I). The millisecondresolution timing of these dynamics are also highly valuable for dissociating the temporal profile of when different cues are processed, when different cognitive processes come into play, or how individual or cross-cultural differences shape processing [37,49,92]. Just as responses involving motor demands in other tasks (e.g., longer reaction times in semantic or evaluative priming, or the IAT) are often interpreted as reflecting implicit processes, there is good reason to suspect that mouse-trajectory dynamics in speeded responses also reflect implicit processes. That said, future research is needed to investigate the more implicit or explicit nature of these dynamics, their boundary conditions, and the extent to which they are controllable. 


\section{Box 1. Figure I. Mouse-tracking measures for understanding cognitive dynamics. A}

"hidden" partial activation of the male category is illustrated, indicated by the hand's attraction to the 'male' category response. Left panel: Magnitude or strength of the activation can be measured by maximum perpendicular deviation (dashed line) or area under the curve (blue highlight) relative to an idealized straight-line response trajectory (examples in Fig. 2A and 2B). Right panel: The stability or instability of category activation dynamics can be measured by the number of abrupt directional changes along the horizontal $x$-axis (axis of decision) (example in Fig. 2C). 
Figure 1, Key Figure. Dynamic Interactive (DI) Model of social perception. (A) A feedforward approach to social perception (top) assumes a target's face is visually encoded by some feature space or set of feature detectors, which thereafter activates related social categories. Those categories then automatically activate related stereotypes, attitudes, and goals, which affect and may be affected by high-level behavior. The DI Model [4] (bottom), posits additional feedback influences inherent to the system, wherein stereotypes, attitudes, and goals constrain and inform category activation and featural representation. In contrast to the feed-forward approach, the DI Model predicts that category activation (e.g., male) and featural representation (e.g., larger jaw) are impacted not only by lower-level visual processing, but also by activated stereotypes (e.g., aggressive) in top-down fashion. Featural representation is simplified for illustration; feature spaces using pixel-based models or computational models of ventral-visual processing may also be implemented. Permission to use the sample face image was obtained [93]. (B) A neural network for flexible split-second social perception, including the fusiform gyrus (FG), orbitofrontal cortex (OFC), and anterior temporal lobe (ATL). The FG is involved in visual processing of faces, and the ATL retrieves social-conceptual associations related to perceived characteristics such as social categories (e.g., stereotypes, person-knowledge). Such social-conceptual information may then be used by the OFC to implement top-down visual predictions that modulate FG representations of faces in line with those predictions. This network supports a rapid and flexible integration of bottom-up facial cues and higher-order social cognitive processes. 
Figure 2. Examples of "hidden" social category activations. In the mouse-tracking tasks, one face was presented at a time at the center of the screen, and participants moved the cursor from the bottom-center into response options at either top corners of the screen. (A) The extent to which participants were partially attracted to the 'male' response when categorizing a female politician's face (i.e., the strength of a partial male category activation) predicted a decreased likelihood of winning her electoral contest, particularly in U.S. conservative states [18]. (B) When categorizing faces' race, low-status attire (e.g., janitor uniform) led trajectories to be partially drawn to the 'Black' response due to stereotypes linking Blacks to low-status. Although not depicted, high-status attire (e.g., business suit) conversely compelled trajectories toward the 'White' response [26]. (C) When categorizing racially-ambiguous faces, White participants in neighborhoods with higher exposure to Black individuals showed more stable co-activation of the White and Black categories. Those in neighborhoods with lower exposure to Black individuals showed more unstable co-activation, indicating by abrupt White-Black category shifting. Additional work suggested these unstable category-activation dynamics were due to low-exposure participants' stereotypes that White and Black individuals are highly dissimilar, which affects perceptions (Fig. 3). The map depicts geolocated participants (black dots) overlaid on U.S. Census data of the concentration of Black population, which was used as a measure of interracial exposure [21]. 
Figure 3. Race perception and interracial exposure. The DI Model treats social categories as attractors. In this illustration, two energy minima indicate the White and Black attractor states in a system in which two forces jointly drive race perception: visual processing (blue arrows) and conceptual knowledge (red arrows). Conceptual knowledge (red arrows) reflects the natural force in the system to minimize energy and descend down the landscape, like a ball's compulsion to roll down a hill. The trajectory of the system (represented by the ball's motion) reflects the process of race perception, where gradual descent into an attractor reflects an increasing activation of that social category and an increasingly confident interpretation that the category is represented in the visual input. When confronted by a racially-ambiguous face, visual processing initially seeks to drive the system toward the ridge in between the attractors, "pushing" the White and Black activations together to be in line with the ambiguous facial input. Conceptual knowledge, on the other hand, seeks to rapidly "pull" the two categories apart, seeking to drive the system into one alternative or the other. When White and Black categories are more conceptually dissimilar (as in White individuals with lower interracial exposure), the attractors will be more differentiated [21], creating a steeper descent and a stronger "pull" to descend into the categories. When low-exposure perceivers are presented with racially-ambiguous faces, visual processing tries to "push" together two categories that conceptual knowledge is trying to strongly "pull” apart, creating more unstable dynamics. Computational simulations and mousetracking findings (Fig. 2C) supported these predictions. Adapted from [21]. 
Figure 4. The inherent intersection of social categories. Illustration of the MVPA representational similarity analysis findings of [39]. At the top left, a dissimilarity matrix (DM) depicts the similarity/dissimilarity in conceptual knowledge of all pairwise gender, race, and emotion categories, based on trait ratings regarding social categories (no faces involved). Blue colors indicate similarity and red colors indicate dissimilarity. For instance, the Black and Angry categories tended to be quite similar, indicating conceptual overlap (bottom left). At the top middle, another DM depicts the similarity/dissimilarity in perceptions based on mouse-tracking data, where similarity indicates bias. For instance, Black and Angry faces tended to elicit similar mouse-tracking patterns, such as a greater bias to perceive Black faces as 'Angry' even when not displaying angry cues (bottom middle). The structure of the conceptual DM significantly predicted the structure of the perceptual DM, which in turn predicted the structure of multi-voxel patterns in the FG (and OFC). Thus, in this example, a greater conceptual overlap between Black and Angry categories predicted a greater bias to perceive faces belonging to those categories similarly (e.g., Black faces as angry), and this in turn predicted a greater similarity in multi-voxel patterns for the Black and Angry categories when viewing such faces in the scanner. 
Figure 5. The many faces of context. The top-down impact of stereotypes on face perception shares a fundamental similarity with more general top-down impacts of context on object or word perception. Cues in the visual periphery of a face or even within a face (e.g., its other category memberships) provide a "context" that may be semantically (i.e., stereotypically) associated with the focal percept. (A) An identical ambiguous object is readily perceived to be a drill when in a garage but to be a hairdryer when in a bathroom. Adapted from [74]. (B) An identical ambiguous $\mathrm{A} / \mathrm{H}$ letter is readily disambiguated as " $\mathrm{A}$ " in the context of "CAT" but "H" in the context of "THE" due to prior knowledge. (C) Contextual attire cues bias perception of a racially-ambiguous face to be White when surrounded by high-status attire but to be Black when surrounded by low-status attire, due to stereotypic associations between race and social status [26]. Adapted from [26]. (D) Contextual cues may be embedded within the face itself, such as when an emotionally ambiguous face is perceived to be angry when male but happy when female, due to stereotypic associations linking men to anger and women to joy [39,54]. 


\section{Outstanding Questions Box}

- Extensive research has explored face-based trait inferences (e.g., perceiving trustworthiness and dominance) and social evaluation (e.g., positive-negative) from faces. Do such perceptions share similar mechanisms as perceiving social categories? How can computational and neural models of perceptions involved in social categorization be integrated with those involved in trait inference and social evaluation?

- Much research outside of social neuroscience has examined the role of the OFC in top-down expectations that constrain visual-object processing, which provides a precedent for understanding how top-down stereotypic expectations may constrain face processing. Other higher-order factors such as attitudes and goals can affect perceptions, but do such top-down effects depend on OFC modulation or other mechanisms?

- The functional relationships between the FG, OFC, and ATL and their precise roles in the context of initial social perceptions remain unclear. How can network modeling, functional connectivity analyses, and other techniques such as MEG clarify the interplay and timing of these regions' participation in flexible social perception?

- At exactly which levels of representation do top-down impacts of social cognitive processes manifest? MVPA combined with mouse-tracking and other sensitive behavioral techniques will likely be important in addressing this question. How can such work inform recent resurgences of the argument for cognitive impenetrability of perception?

- The flexibility and bias in initial social perceptions can be considered both adaptive (facilitation by context and expectations that streamlines processing; motivational biases to self-protect) or maladaptive (perceptual errors and sub-optimal performance). What are the evolutionary origins of such top-down effects on perception: Do they represent an adaptation, or a byproduct of more general perceptual-cognitive interactions (or some other process)? 
A

Feed-Forward Approach
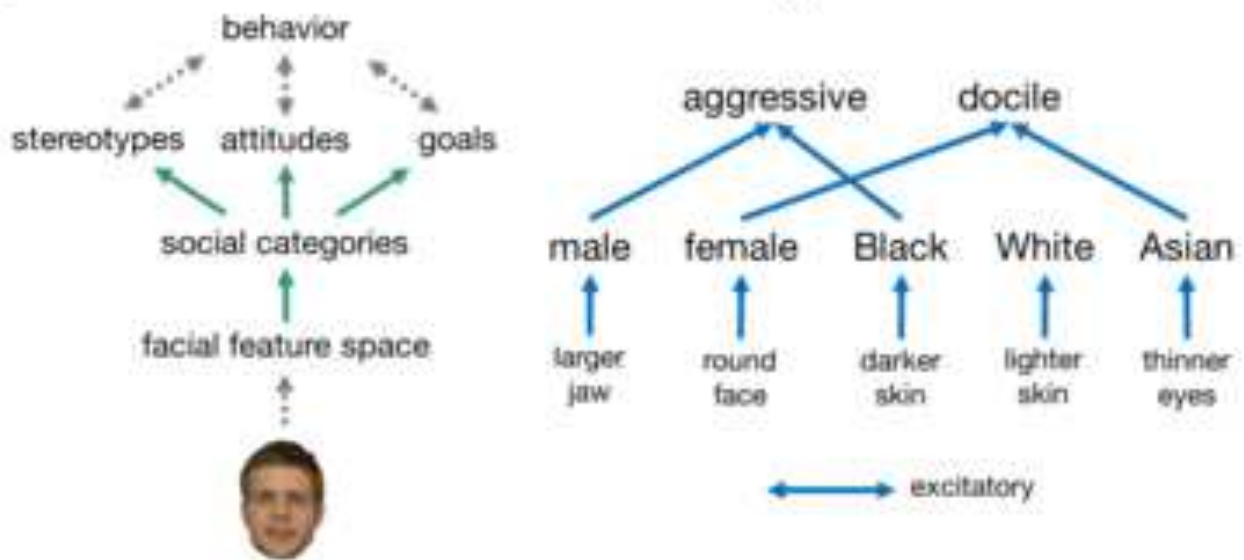

Dynamic Interactive Model

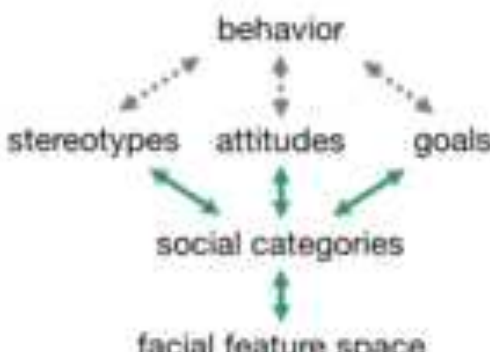

facial feature space
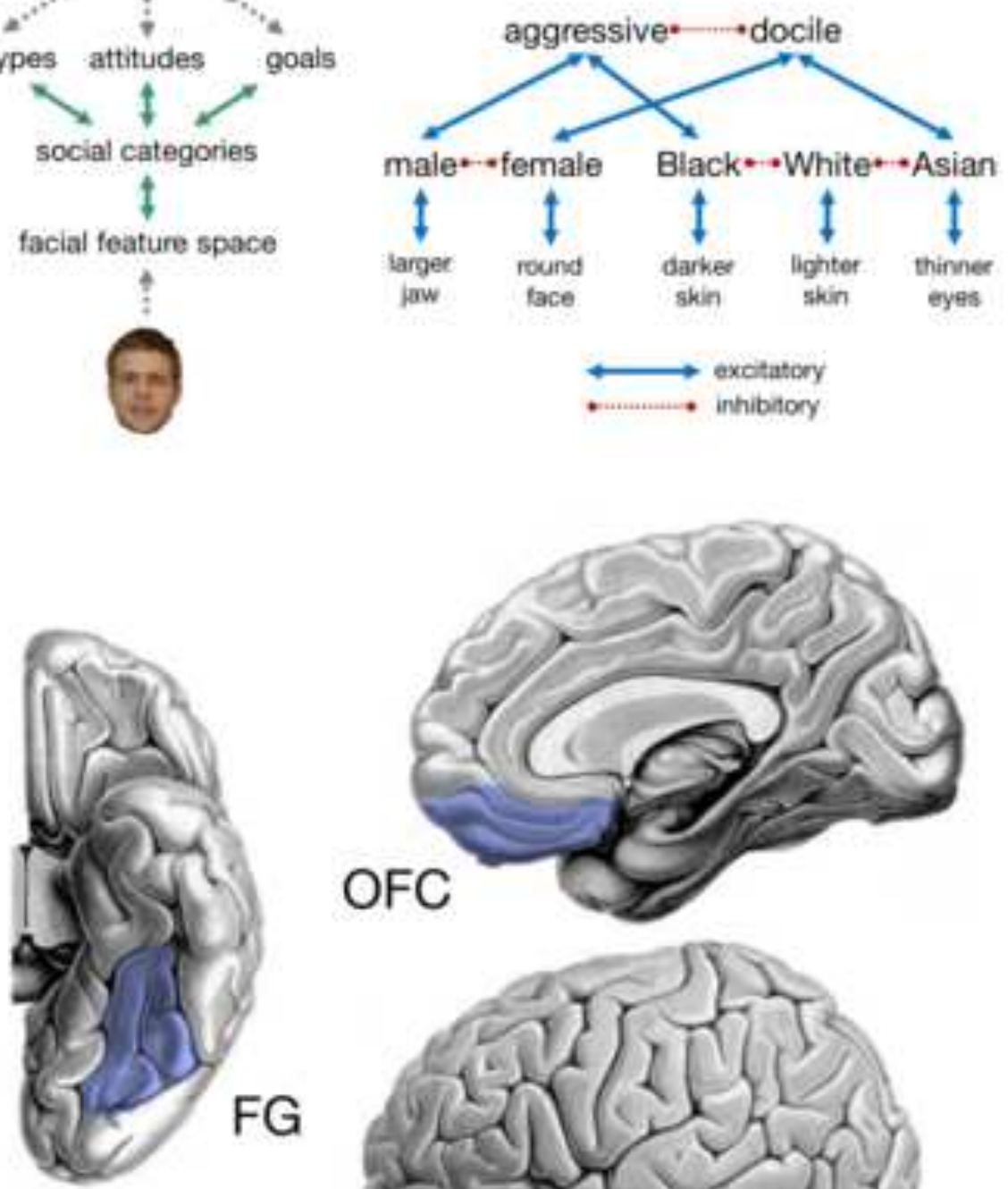

FG

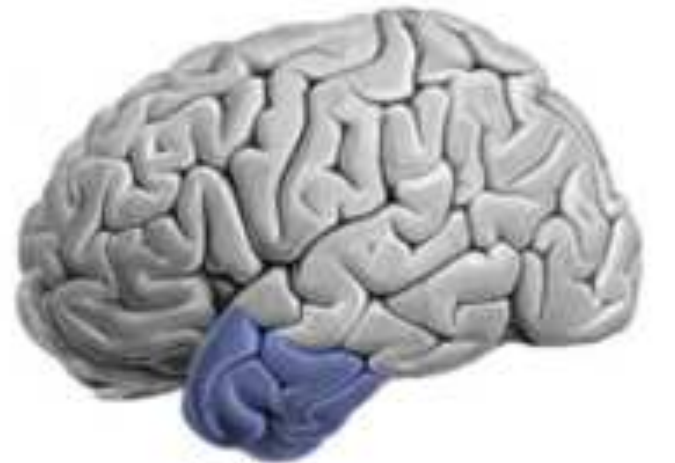

ATL 


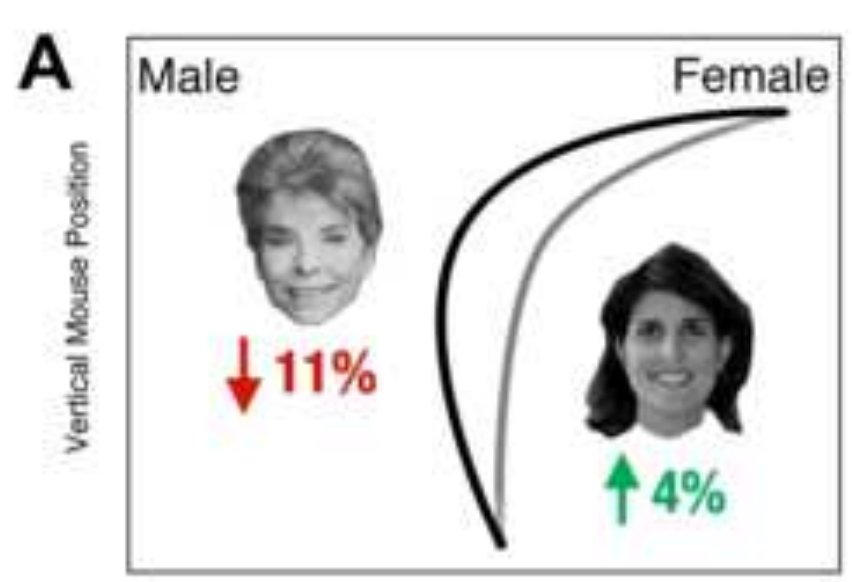

Horizontal Mouse Position
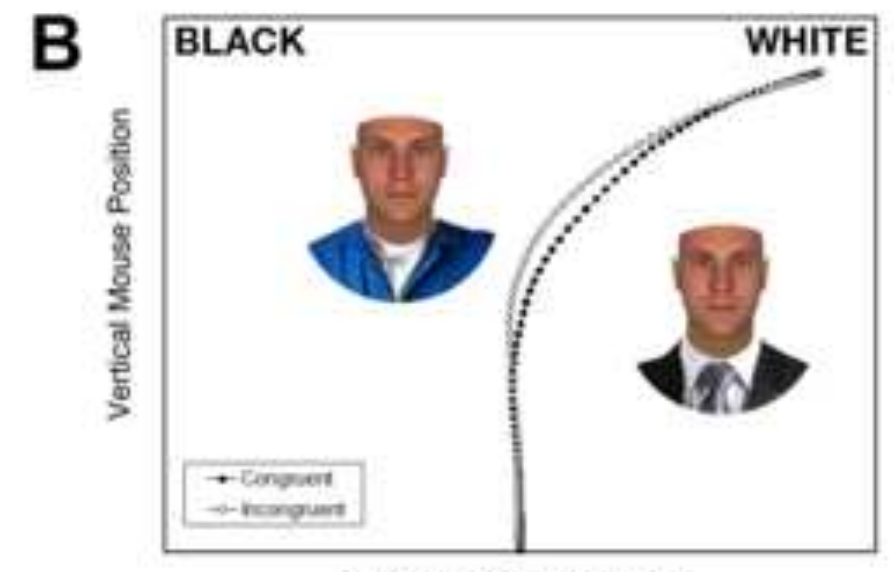

Horizontal Mouse Position

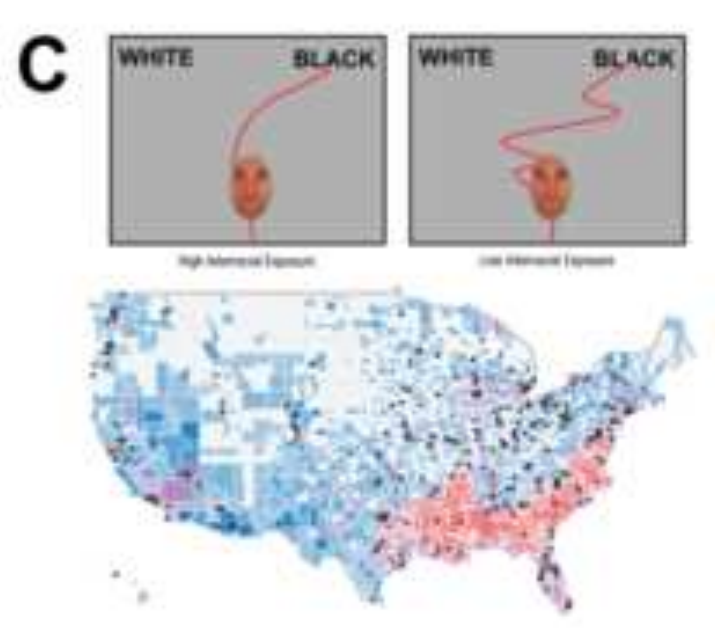


A

$-$

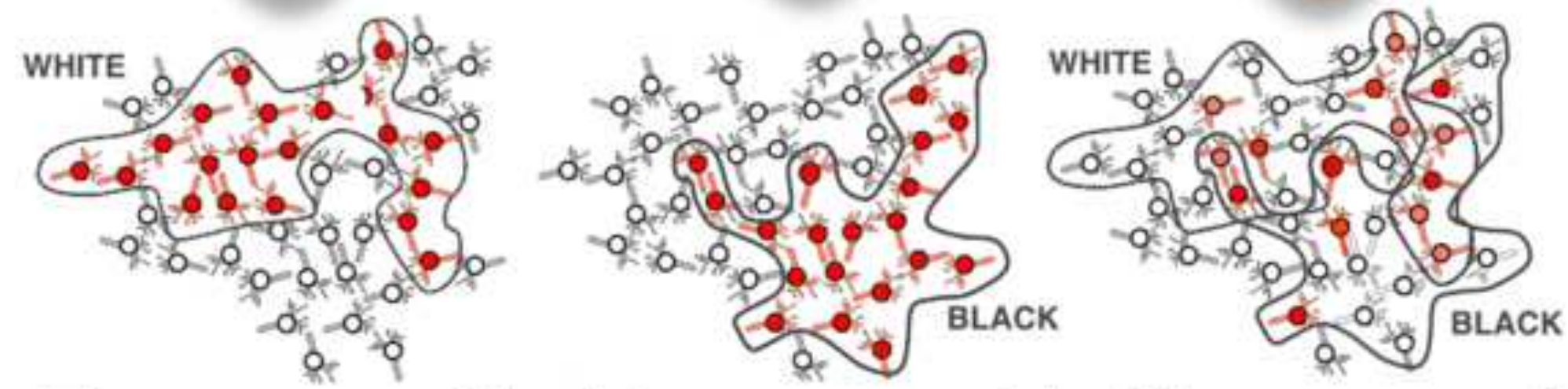

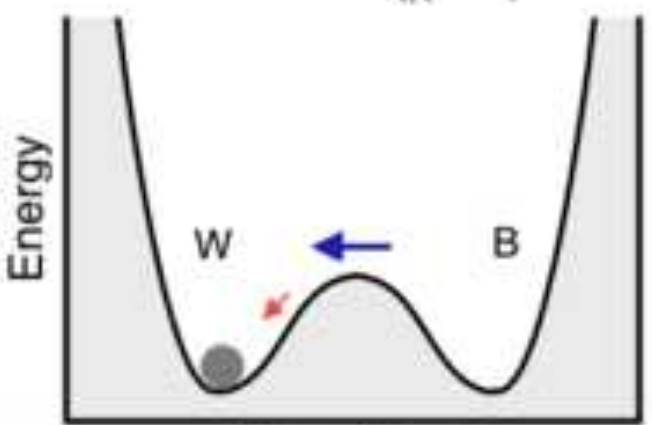

Network state

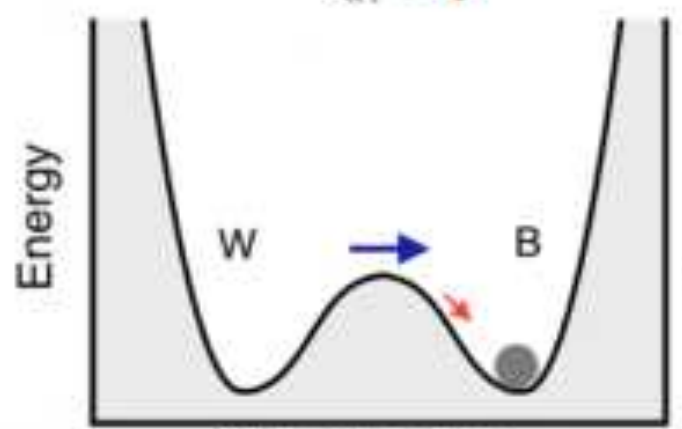

Network state
B

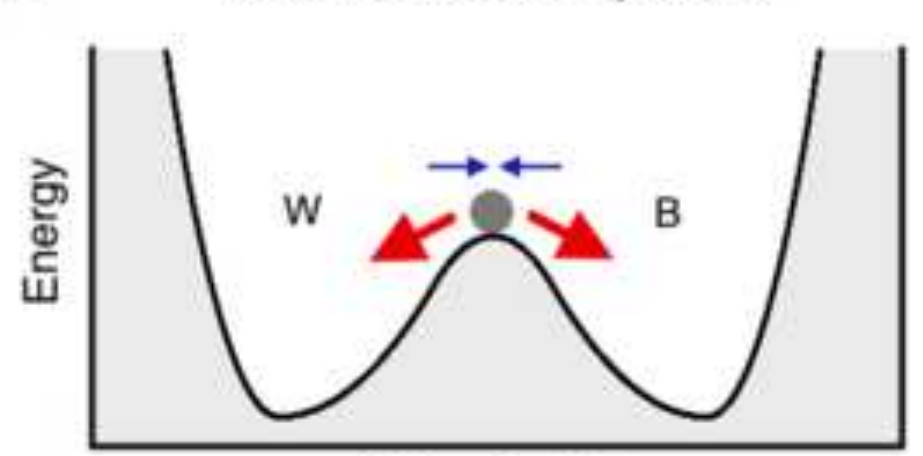

Network state

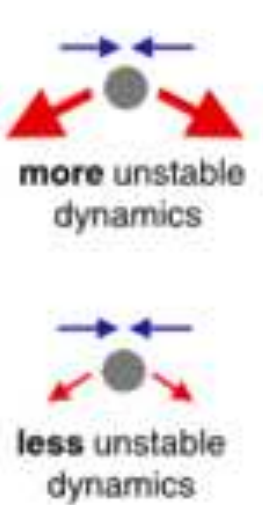

dynamics

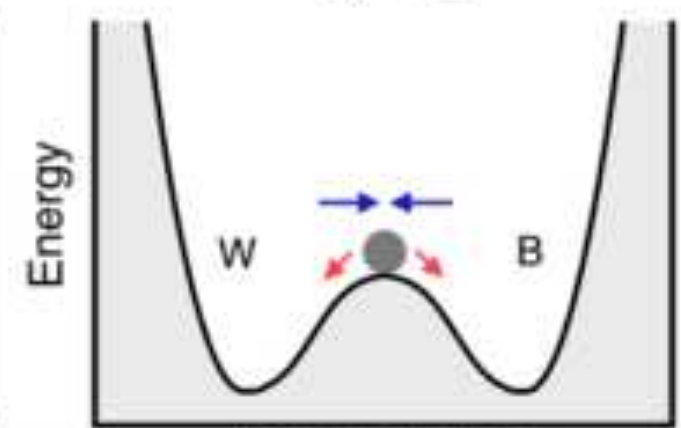

Network state

high interracial exposure

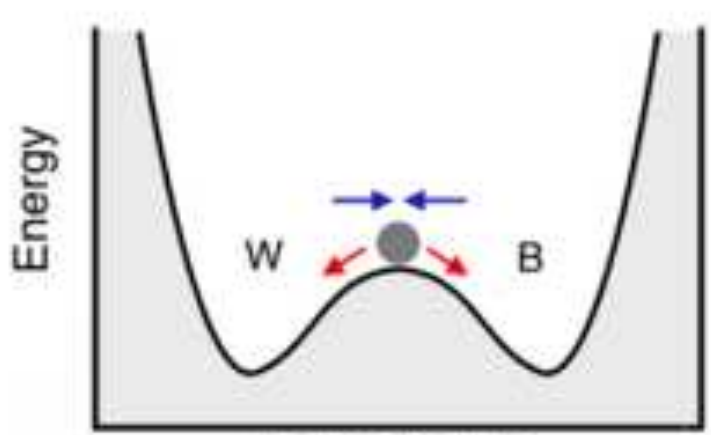

Network state 


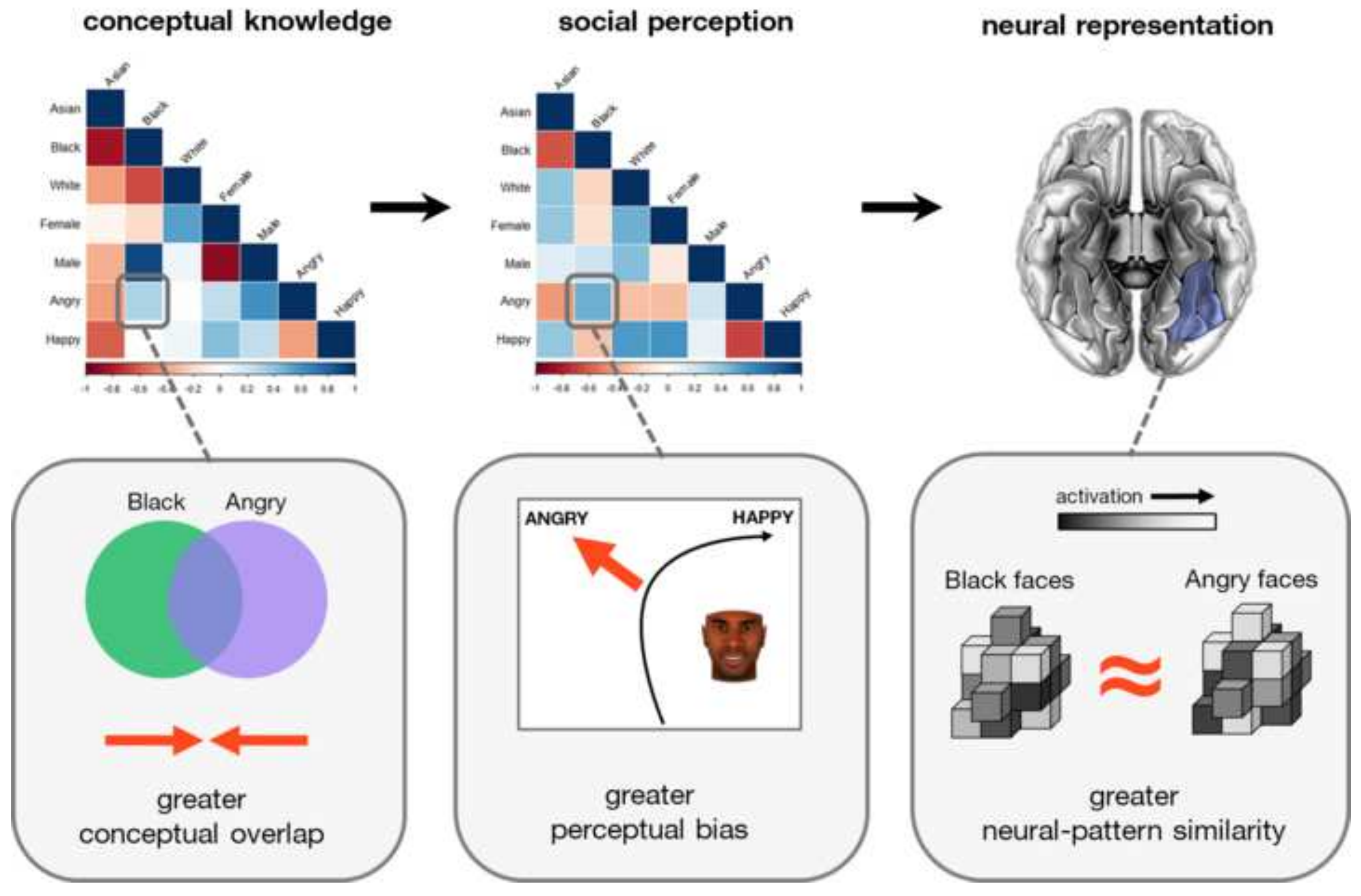



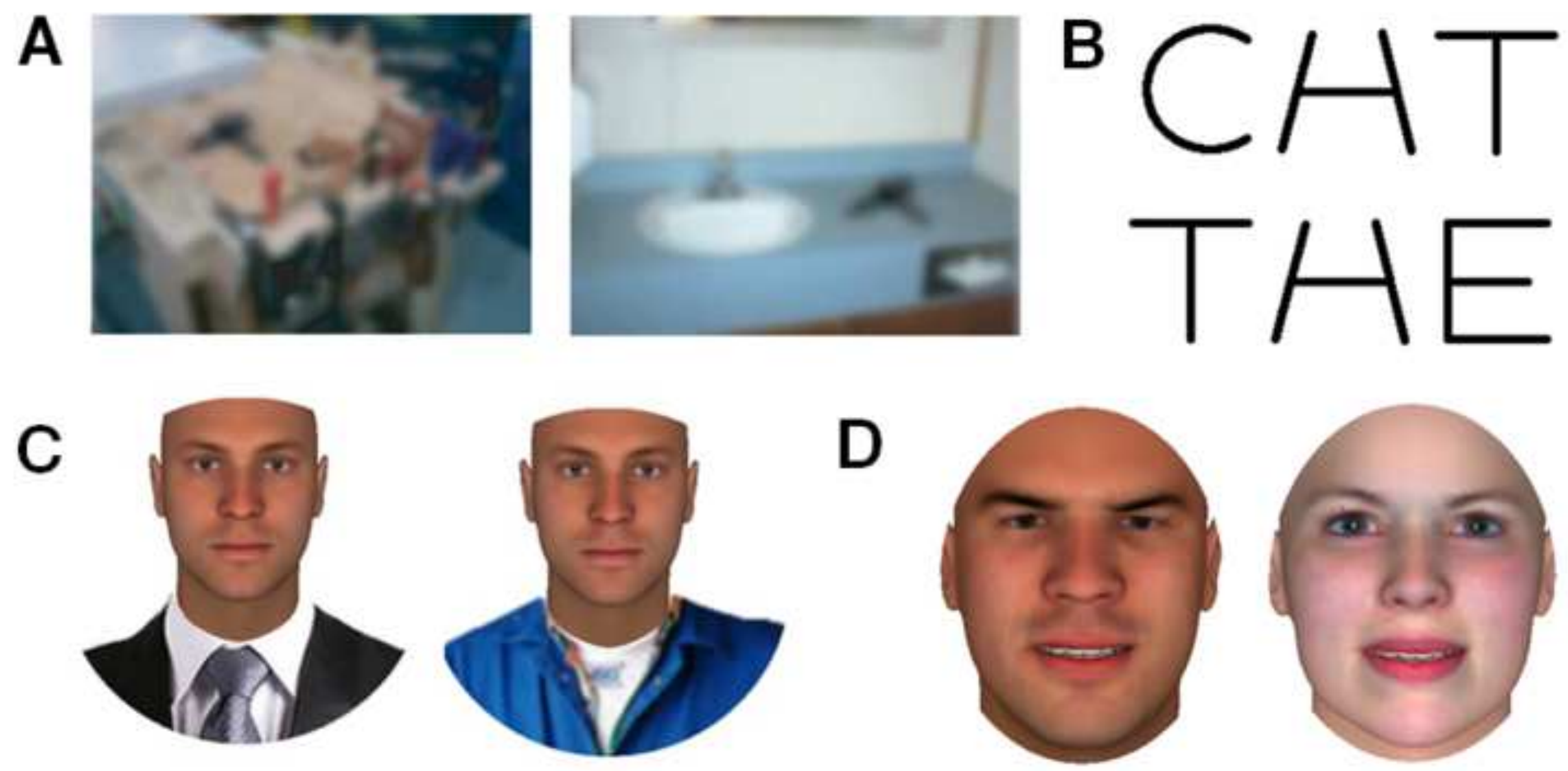
A

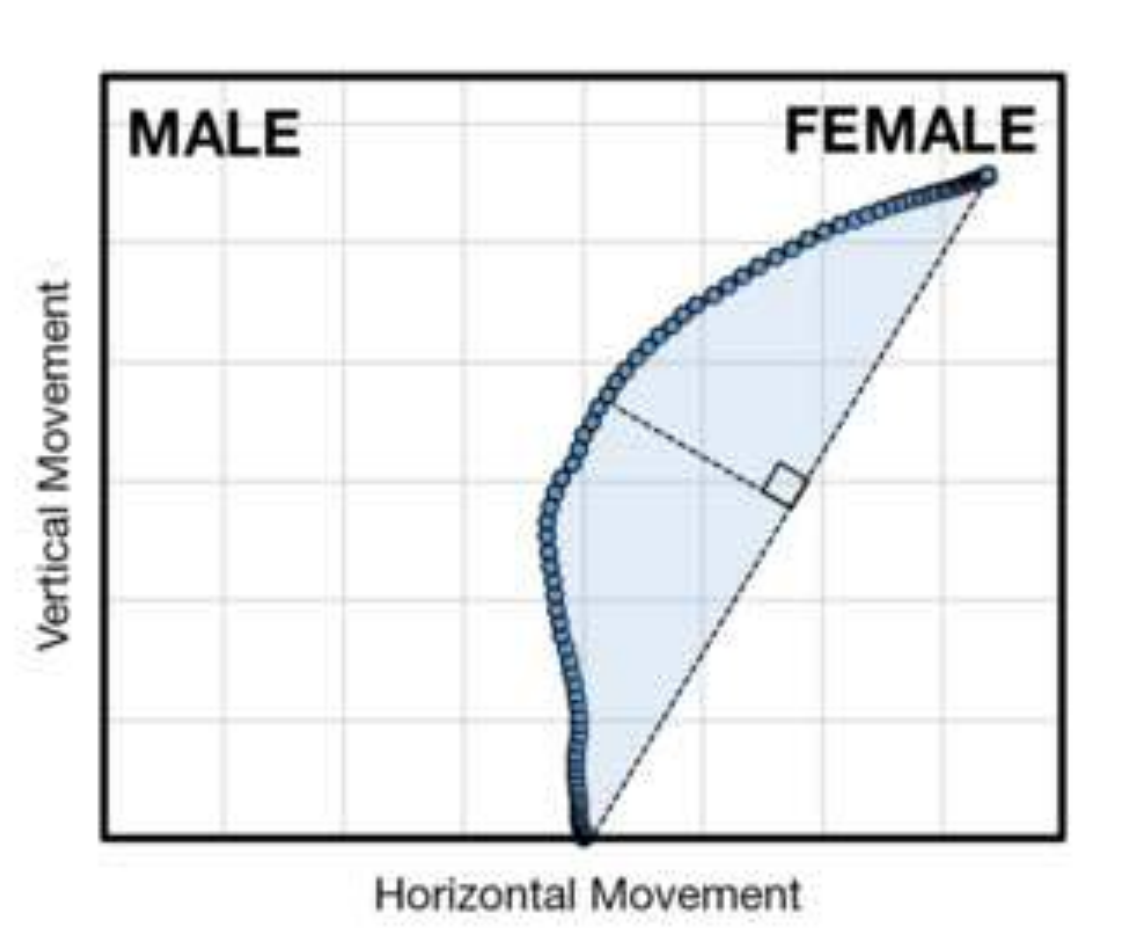

Horizontal Movement

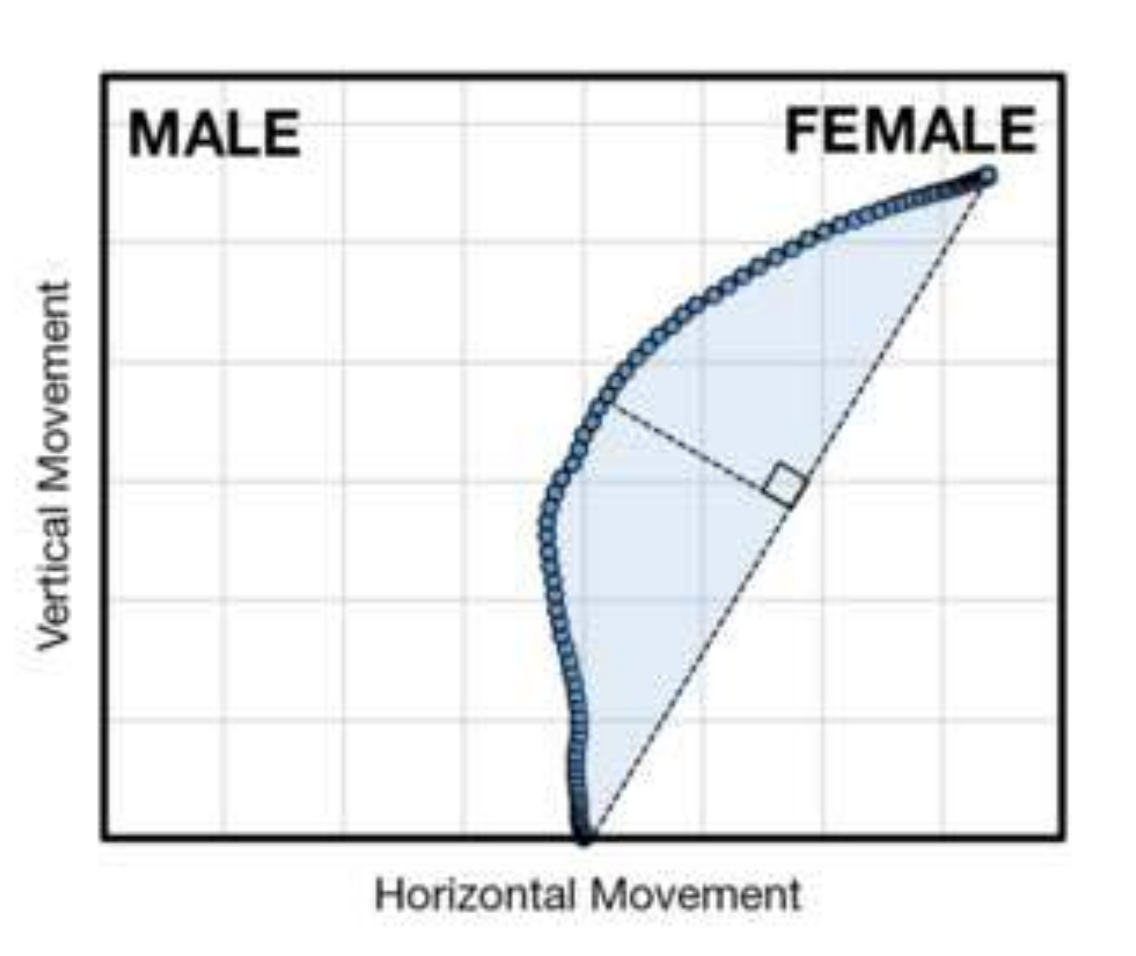

Horizontal Movement

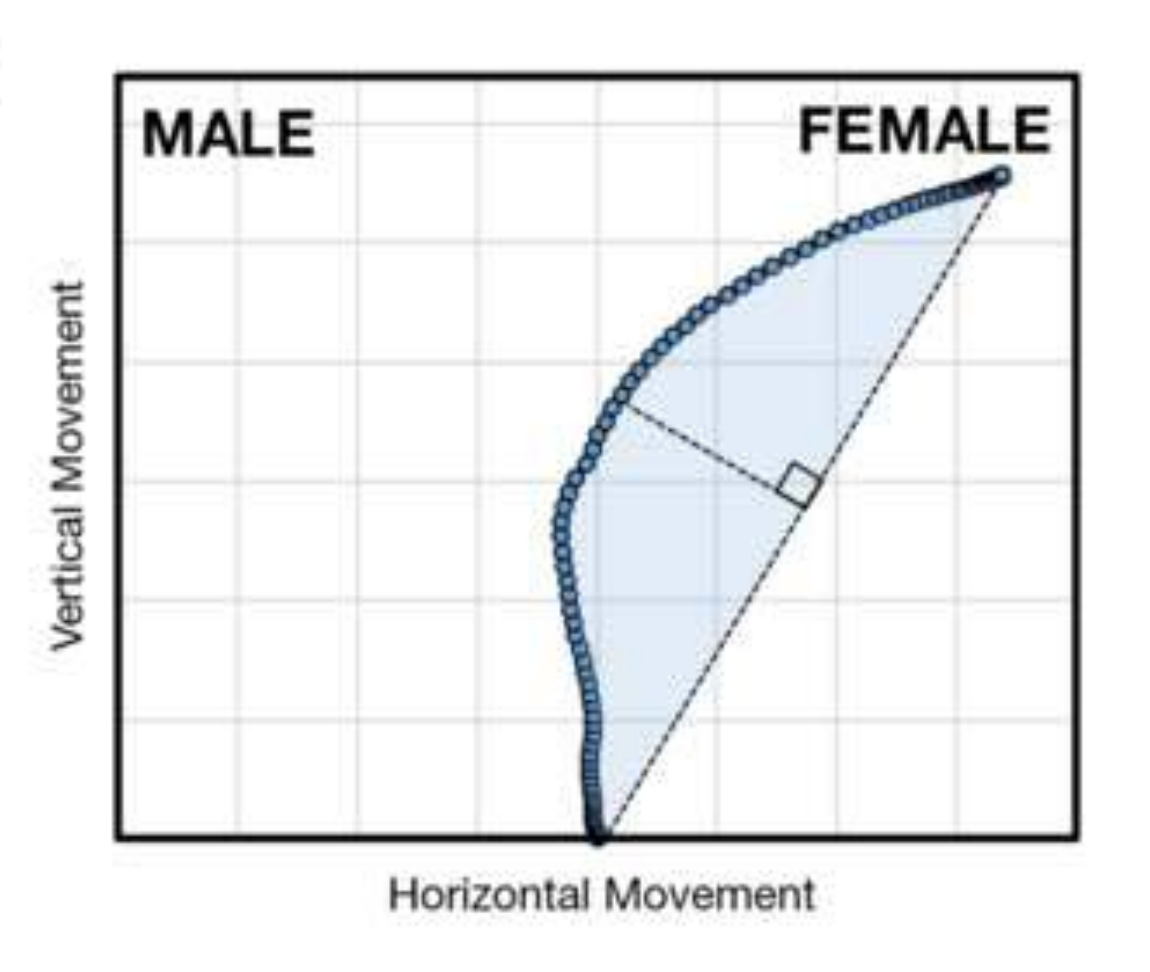

Horizontal Movement

Chorizontal Movement
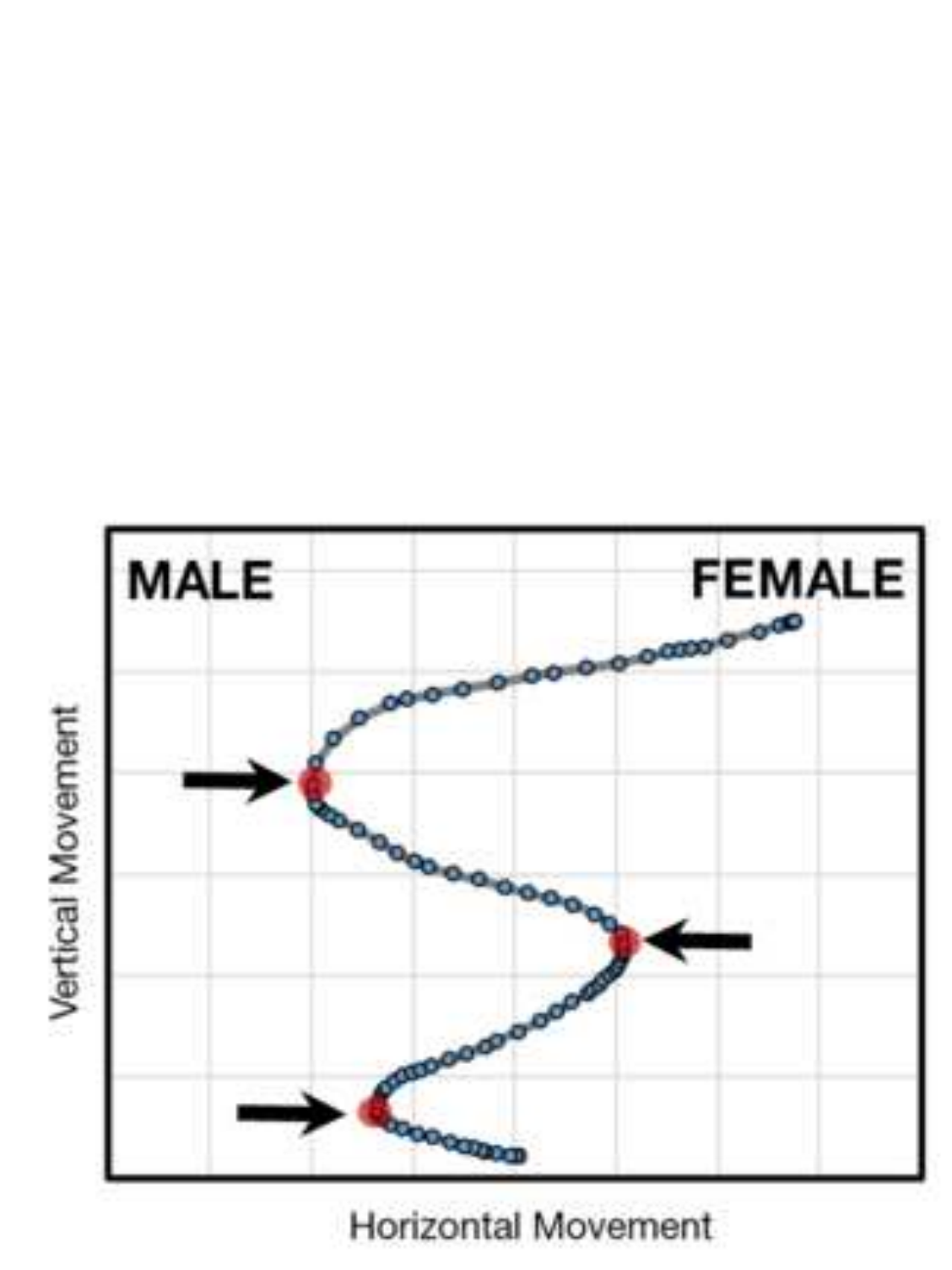